\title{
WORKING GROUP ON MAGNETOGRAPH CALIBRATION
}

The working group convened during the Paris IAU Symposium on Solar Magnetic Fields. It was decided that the comparison of magnetograms obtained at different observatories should proceed in two stages. First a pilot program was to be run including only observatories in the southwestern United States (Mt. Wilson, Kitt Peak, High Altitude Observatory and Sacramento Peak); based on the experience of this pilot program a second stage was to be planned to include all observatories. The magnetograph parameters were defined and it was decided to use both the $\lambda 5250$ and $\lambda 6303$ lines. The pilot program was run in October 1970; observations were obtained only at Kitt Peak and Mt. Wilson. Comparison showed almost a factor of 2 difference in the field measurements between these two observatories. Since then this factor has been brought closer to unity by improved calibration methods. The full second stage was run in October 1972. The table below shows a comparison between weak magnetic field amplitudes for October 21, 1971 for all observatories which obtained data in this period. The $\lambda 5250$ observations are in exceptionally good agreement, deviations being less than $\pm 25 \%$ from the mean. The $\lambda 6303$ observations disagree greatly, however. Many prominent observatories with magnetographs were unable to take part because of cloud conditions. A second global attempt undertaken jointly with the CINOF campaign in June 1972, also failed to include these observatories.

Continuation of the program will be considered at the Sydney General Assembly.

Table 1. Scaling factors for weak magnetic fields $(H<350 \mathrm{G})$. Kitt Peak $\lambda \mathbf{5 2 5 0}$ observations on October 21, 1971, are taken as unit values

$\begin{array}{lccc}\text { Observatory } & \begin{array}{c}\text { Julian Date } \\ (2441240+)\end{array} & \text { Line } & \text { Scaling Factor } \\ \text { Kitt Peak } & 6.24 & \lambda 5250 & \\ \text { Kitt Peak } & 5 \cdot 25 & \lambda 5250 & 1.00 \\ \text { Sac. Peak } & 6.09 & \lambda 5250 & 0.81 \\ \text { Mt. Wilson } & 6.37 & \lambda 5250 & 1.32 \\ \text { Irkutsk } & 5.76 & \lambda 5250 & 0.83 \\ \text { Capri } & 5 \cdot 79 & \lambda 5250 & 1.08 \\ \text { Meudon } & 6.06 & \lambda 5250 & 1.12 \\ \text { Sac. Peak } & 6.11 & \lambda 6303 & 1.16 \\ \text { Capri } & 5.89 & \lambda 6303 & 1.66 \\ \text { Kitt Peak } & 6.19 & \lambda 5233 & 0.73 \\ \text { Kitt Peak } & 6.27 & \lambda 5253 & 1.62 \\ \end{array}$

B October $21,19710.00^{\mathrm{m}}$ UT $=2441245 \cdot 50$ Julian Date

J. M. BECKERS

Chairman of the Working Group

\section{BIBLIOGRAPHY}

Abdussamatov, H. I. 1971, IAU Symp. 43, p. 231.

Acton, L. W., Catura, R. C., Culhane, J. L. 1972a, Space Research XIII, in press.

Acton, L. W., Catura, R. C., Meyerott, A. J., Wolfson, C. J., Culhane, J. L. $\quad$ 1972b, Solar Phys. $26,183$.

Adam, M. G. $\quad 1971$, Mon. Not. R. astr. Soc. 155, 169.

Alekseyev, I. I., Shabanskiy, V. P., Shister, A. R. 1971, Geomagnetism and Aeronomy 11, 201.

Allen, C. W. 1971, Phil. Trans. Roy. Soc.-A. 270, 71.

Allum, F. R., Palmeira, R. A. R., Rao, U. R., McCracken, K. G., Harries, J. R., Palmer, I. 1971, Solar Phys. 17, 241.

Altschuler, M. D., and Newkirk, G. $\quad$ 1969, Solar Phys. 9, 131.

Altschuler, M. D., Newkirk, G., Jr., Trotter, D. E., Howard, R. $\quad$ 1971, IAU Symp. 43, p. 588. 
Alvarez, H., Haddock, F., Lin, R. P. $\quad$ 1972, Solar Phys. 26, 468.

Ambroz, P., Bumba, V., Howard, R., and Sykora, J. 1971 , in Solar Magnetic Fields, R. Howard, ed., ed., p. 696 (Reidel, Dordrecht).

Anderson, K. A. 1969, Solar Phys. 6, 111.

Anderson, K. A. 1972, Paper presented at the XIV Meeting of COSPAR, Madrid.

Anderson, K. A., Kane, S. R., and Lin, R. P. 1970, Paper presented at the Seminar on Cosmic Ray Generation, Leningrad, December 1970.

Anisimov, M. M., $\quad$ 1970, Acta Phys. Acad. Scient. Hung. 29, Suppl. 1, 309.

Anzer, J., and Tandberg-Hanssen, E. $\quad$ 1970, Solar Phys. 11, 61.

Argo, H. V., Bergey, J. A., Evans, W. D. $\quad$ 1970, Astrophys. J. 160, 283.

Armstrong, J. W., Coles, W. A. 1972, J. Geophys. Res. 77, 4602.

Armstrong, T. P., Krimigis, S. M., Behannon, K. W. 1970, J. Geophys. Res. 75, 5980.

Armstrong, T. P., Krimigis, S. M., Reames, D. V., and Fichtel, C. E. $\quad$ 1972, J. Geophys. Rev. $77,3607$. Arnoldy, R. 1971, J. Geophys. Res. 76, 5189-5201.

Axisa, F., Avignon, Y., Martres, M. J., Pick, M., and Simon, P. $\quad$ 1971, Solar Phys. 19, 110.

Bachmann, G., Pflug, K., Staude, J. 1970, Solar Phys. 15, 113.

Balasubrahmanyan, V., and Venkatesan, D. 1971, Solar Phys. 19, 257.

Barletti, R., and Tagliaferri, G. L. $\quad$ 1969, J. Atmos. Terr. Phys. 31, 631.

Barletti, R., Tagliaferri, G. L. $\quad$ 1972, Memorie Della Societa Astronomica Italiana XLII, 301.

Ballario, M. C. 1970a, Report UAG-8, 231-238.

Ballario, M. C. 1970b, Ann. de Geophys. 26, 459.

Ballario, M. C. 1972a, Memorie Della Societa Astronomica Italiana XLIII, 55.

Ballario, M. C. 1972b, World Data Center A Report.

Ballif, J. R., Jones, D. E., Skousen, E. N., and Smith, D. T. $\quad$ 1971, J. Geophys. Res. 76, 8401.

Bame, S. J., Asbridge, J. R., Hundhausen, A. J., Montgomery, M. D. $\quad$ 1970, J. Geophys. Res. 75, 6360.

Barker, M. C., and Hatton, C. J. 1971 , Planet. Space Sci. 19, 549.

Batstone, R. M., Evans, K., Parkinson, J. H., Pounds, K. A. 1970 , Solar Phys. 13, 389.

Battacharya, J. C., and Sundes, R. S. 1970, Indian J. Pure Applied Phys. 8, 544.

Beckers, J. M., and Schultz, R. B. 1972, Solar Phys. 27, 61.

Beigman, I. L., Vainshtein, L. A., Vinogradov, A. 1969a, Astron. Zh. 13, 985.

Beigman, I. L., Grineva, Yu. I., Mandelshtam, S. L., Vainhstein, L. A., and Zhitnik, I. A. 1969 b, Solar Phys. 9, 160.

Beigman, I. L., Vainshtein, L. A., Vasil'ev, B. N., Zhitnik, I. A., Ivanov, V. D., Korneev, V. V., Krutov, V. V., Mandelshtam, S. L., Tindo, I. P., and Shurygin, A. I. 1971, Kosmicheskie Issledovaniya 9, 123.

Belcher, J. W., and Davis, L., Jr. 1971, J. Geophys. Res. 76, 3534.

Bertsch, D. L., Fichtel, C. E., and Reames, D. V. 1969, Astrophys. J. (Letters) 157, L53.

Bertsch, D. L., Fichtel, C. E., Reames, D. V. 1972a, Astrophys. J. 171, 169.

Bertsch, D. L., Fichtel, C. E., Pellerin, C. J. 1972b, 'Measurements of the Fe-Group Abundances in Energetic Solar Particles', to be published in Astrophys. $J$.

Bhandari, N., Bhat, S., Lal, D., Rajagopalan, G., Tamhane, A. S., and Venkatavaradan, V. S. 1971, Proc. of the Second Lunar Science Conference 3, 2611.

Bhatnagar, A., and Tanaka, K. 1972, Solar Phys. 24, 87.

Blake, R. L., and House, L. L. 1971, Astrophys. J. 166, 423.

Blocker, N. K., Chambers, W. H., Fehlau, P. E., Fuller, J. C., Kunz, W. E., and Milkey, R. W. 1971 , Solar Phys. 20, 400.

Blumenthal, G. R., Drake, G. W. F., and Tucker, W. H. 1972, Astrophys. J. 172, 205.

Bohlin, J. D., Koomen, M. J., and Tousey, R. 1972, Solar Phys. 21, 408.

Bowman, G. G. 1971, Nature Phys. Sci. 229, 117.

Boyer, R., Henoux, J. C., Sotirovski, P. $\quad$ 1971, Solar Phys. 19, 330.

Branch, D. 1970 , Astrophys. J. 159, 39.

Bräuninger, H., Einighammer, H. J., Feitzinger, J. V., Fink, H. H., Höhn, D. H., Koops, H., Krämer, G., Mayer, U., Möllenstedt, G., and Mozer, M. 1971, Solar Phys. 20, 81.

Bray, J. R. $\quad 1970$, Science 168, 571.

Bray, J. R. 1971, Science 171, 1242.

Brinkman, A. C., and Shaw, M. L. $\quad$ 1972, Solar Phys. 23, 120.

Broadfoot, A. L. 1972, Astrophys. J. 173, 681.

Brown, J. C. 1971, Solar Phys. 18, 489.

Brown, J. C. 1972, Solar Phys. 25, 158. 
Brueckner, G. E. 1972a, Proceedings of the Conference on Flare-Produced Shock Waves in the Corona, National Center for Atmospheric Research, Boulder, Colo., 11-14 Sept., 1972, in press.

Brueckner, G. E. 1972b, Bull. A. A. S. 4, II, 378.

Bruns, A. V., Prokofiev, V. K., and Severny, A. B. $\quad$ 1970, IAU Symp. 36, p. 256.

Bruzek, A. 1972a, Solar Phys. 24, 118.

Bruzek, A. $\quad 1972 b$, Solar Phys. 26, 94.

Bruzek, A., and DeMastus, H. 1970, Solar Phys. 12, 447.

Bruzek, A., and Kuperus, M. 1972, Solar Phys. 24, 3.

Buckley, R. 1971, Planet. Space Sci. 19, 421.

Budyko, M. I. 1969, Tellus 21, 611.

Bumba, V., Krivsky, L., and Sykora, J. 1972, Bull. Astron. Inst. Czech. 23, 85.

Burger, M., Dijkstra, J. H. 1972, Solar Phys. 24, 395.

Burlaga, L. F. 1971, Space Sci. Rev. 12, 600.

Burlaga, L. F., Ogilvie, K. W., Fairfield, D. H., Montgomery, M. D., Bame, S. J. 1971, Astrophys, J. $164,137$.

Burlaga, L. F., Chao, J. K. $\quad$ 1971, J. Geophys. Res. 76, 7516.

Bureau, R. A., and Craine, L. B. 1970, Nature 228, 984.

Burns, J. 1970, Ph.D. Thesis, Univ. of Hawaii.

Burton, W. M., Ridgeley, A. 1970, Solar Phys. 14, 3.

Cantu, A., Godoli, G., and Poletto, G. 1970, Solar Phys. 15, 356.

Carlqvist, P. 1972, Cosmic Electrodyn 3, 377.

Castelli, J. P., and Richards, D. W. 1971, J. Geophys. Res. 76, 8409.

Cattaneo, M. B., Formisano, V., Moreno, G., Palmiotto, F., Palutan, F., Saraceno, P. $\quad$ 1971, Solar Phys. 17, 468.

Chao, J. K., and Goldstein, B. 1972, J. Geophys. Res. 77, 5455.

Cheng, Chung-Chieh. 1972b, Space Sci. Rev. 13, 3.

Chernosky, E. 1966, J. Geophys. Res. 71, 965.

Chin, Y. C., Lusignan, B. B., and Fung, P. C. W. 1971, Solar Phys. 16, 135.

Chubb, T. A. 1972, Solar-Terrestrial Physics, Part I., de Jager, ed.

Chupp, E. L. $\quad$ 1971, Space Sci. Rev. 12, 486.

Chupp, E. L., Forrest, D. J., Higbie, P. R., Suri, A. N., Tsai, C., Dunphy, P. P. $\quad$ 1972, Nature Phys. Sci., in press.

Coleman, P. J., Jr., and Rosenberg, R. L. $\quad$ 1971, J. Geophys. Res. 76, 2917.

Coles, W. A., and Maagoe, S. $\quad$ 1972, J. Geophys. Res. 77, 5622.

Coppi, B., and Friedland, A. B. 1971, Astrophys. J. 169, 379.

Cortellessa, P., di'Benedetto, P., and Paizis, C. 1970, Solar Phys. 14, 427.

Cortellessa, P., di'Benedetto, P., and Paizis, C. $\quad$ 1971, Solar Phys. $20,474$.

Coupiac, P., and Koutchmy, S. 1972, Astron. Astrophys. 16, 272.

Cowley, C., and Marlborough, J. M. 1969, Astrophys. J. 158, 803.

Crawford, H. C., Price, P. B., and Sullivan, J. D. 1972 , to be published.

Croft, T. A. 1971, Radio Science 6, 55.

Cronyn, W. M. 1972, Astrophys. J. 171, L101.

Croom, D. L. 1971, Solar Phys. 19, 152.

Croom, D. L., and Powell, R. J. $\quad$ 1971, Solar Phys. 20, 136.

Crozaz, G., and Walker, R. M. 1971, Science 171, 1237.

Culhane, J. L. $\quad$ 1969, Mon. Not. R. astr. Soc. 144, 375.

Culhane, J. L., Sanford, P. W., Shaw, M. L., Phillips, K. J. H., Willmore, A. P., Bowen, P. J., Pounds, K. A., and Smith, D. G. 1969, Mon. Not. R. astr. Soc. 145, 435.

Culhane, J. L., and Acton, L. W. 1970, Mon. Not. R. astr. Soc. 151, 141.

Culhane, J. L., and Phillips, K. J. H. 1970a, Solar Phys. 11, 117.

Culhane, J. L., and Phillips, K. J. H. 1970 , Astrophys. J. 160, 309.

Culhane, J. L., Vesecky, J. F., and Phillips, K. J. H. 1970, Solar Phys. 15, 394.

Cuny, Y. 1971, Solar Phys. 16, 293.

Daigne, G., Lantos-Jarry, M. F., and Pick, M. 1971, IAU Symp. 43, p. 609.

Daigne, G. 1971, in Solar Magnetic Fields, R. Howard, ed., p. 376 (Reidel, Dordrecht).

Das Gupta, M. K., and Sarkar, S. K. $\quad$ 1971, J. Roy. Astron. Soc. Canada 65, 66.

Datlowe, D., L'Heureux, J., and Meyer, P. 1970, Electrons from Solar Flares in the 10 to $200 \mathrm{MeV}$ region, Enrico Fermi Institute, EFI 69-86. 
Datlowe, D. 1971, Solar Phys. 17, 436.

Datlowe, D. 1972, J. Geophys. Res. 77, 5374.

Davis, L., Jr. 1972, Solar Terrestrial Physics/1970: Part II, Dyer, ed., p. 32 (Reidel, Dordrecht).

De Feiter, L. D. 1971, Solar Phys. 19, 207.

De Feiter, L. D., and Śvestka, Z. 1972, Space Sci. Rev. 13, 824.

De Jager, C. 1967, Solar Phys. 2, 327.

De Jager, C. $\quad$ 1972, in Solar Terrestrial Physics, Part I, De Jager, ed,, p. 1 (Reidel, Dordrecht).

DeMastus, H. L., Wagner, W., and Robinson, R. D. 1972, Proceedings of the Conference on Flare-

Produced Shock Waves in the Corona, National Center for Atmospheric Research, Boulder, Colo., 11-14 Sept. 1972, in press.

Deshpande, S. D., and Subrahmanyam, C. V. $\quad$ 1971, J. Atmos. and Terres. Phys. 34, 211.

Deubner, F. L., and Göhring, R. 1970, Solar Phys. 13, 118.

Dicke, R. H. 1970, Astrophys. J. 159, 25.

Dilworth, C., Maccagni, D., Perotti, F., Tanzi, E. G., Mercier, J. P., Raviart, A., Treguer, L., and Gros, M. 1972, Solar Phys. 23, 487.

Dodson, H. W., and Hedeman, E. R. 1970, Solar Phys. 13, 401.

Dodson, H. W., and Hedeman, E. R. 1971, Report UAG-14, WDC-A.

Dodson, H. W., and Hedeman, E. R. 1972a, Bull. A. A. S. 4, 309.

Dodson, H. W., and Hedeman, E. R. 1972b, Bull. A. A. S. 4, 380.

Dodson, H. W., Hedeman, E. R., and Miceli, M. R. 1972, Solar Phys, 23, 360.

Dolginova, Y. N., and Korchak, A. A. 1968, Soln. Dann. 6, 81.

Donnelly, R. F. $\quad$ 1969, Astrophys. J. 158, L165.

Donnelly, R. F. $\quad$ 1971, Solar Phys. 20, 188.

Dorman, L. I., and Inozentzeva, O. I. 1969, Izv. Ak. Nauk. SSSR, 1734-1741, Serfiz. 33, 1898-1907.

Dorman, L. I., Kaminer, N. S., and Kebuladze, T. V. $\quad$ 1970, Ak. Nauk. SSSR Izv. 32, (trans.) $2104-2113$.

Dorman, L. I. $\quad$ 1970, Ak. Nauk. SSSR Bull. Phys. Ser. 34, 2071-2085 (Trans. All-Union Conf. on CosmicRay Phys., Leningrad, Oct. 1969).

Doschek, G. A. 1971, Review paper presented at The Third Symp. on Ultraviolet and X-Ray Spectroscopy, Utrecht.

Doschek, G. A. 1972, Space Sci. Rev., 13, 765.

Doschek, G. A., and Meekins, J. F. 1970, Solar Phys. 13, 220.

Doschek, G. A., Meekins, J. F., Kreplin, R. W., Chubb, T. A., and Freidman, H. 1971a, Astrophys. J. 164, 165.

Doschek, G. A., Meekins, J. F., Kreplin, R. W., Chubb, T. A., Freidman, H. 1971b, Astrophys. J. $170,573$.

Doschek, G. A., Meekins, J. F., Kreplin, R. W., and Chubb, T. A. 1972, Space Research XII, in press.

Drake, J. F., Gibson, J., and Van Allen, J. A. 1969, Solar Phys. 10, 433.

Drake, J. F. $\quad$ 1971, Solar Phys. 16, 152.

Dryer, M. 1970, Cosmic Electrodynamics 1, 348.

Dryer, M., Smith, Z. K., Endrud, G. H., and Wolfe, J. H. $\quad$ 1972, Cosmic Electrodynamics 3, 184.

Dulk, G. A., and Altschuler, M. D. 1971, Solar Phys. 20, 438.

Dunckel, N., Helliwell, R. A., and Vesecky, J. 1972, Solar Phys. 25, 197.

Dunn, R. B. $\quad 1970$, Bull. A. I. A. A. 7, 564.

Dunn, R. B. 1971a, IAU Symp. 43, p. 65.

Dunn, R. B. 1971b, in Physics of the Solar Corona, C. Macris, ed., p. 106, (Reidel, Dordrecht).

Dupree, A. K., and Reeves, E. M. 1971, Astrophys. J. 165, 599.

Elgaroy, O., and Lyngstad, E. 1972, Astron. Astrophys. 16, 1.

Elliott, H. $\quad$ 1972, in Solar Terrestrial Physics, Part I, De Jager, ed., p. 134 (Reidel, Dordrecht).

Elwert, G., and Haug, E. 1970, Solar Phys. 15, 234.

Elwert, G., and Haug, E. 1971, Solar Phys. 20, 413.

Engvold, O. 1972a, Astron. Astrophys. Suppl., in press.

Engvold, O. 1972b, Solar Phys. 23, 346.

Engvold, O., Moe, O. K., and Maltby, P. $\quad$ 1970, Astron. Astrophys. 9, 79.

Engvold, O., and Livingston, W. 1971, Solar Phys. 20, 375.

Enomé, S., Kakinuma, T., and Tanaka, H. $\quad$ 1969, Solar Phys. 6, 428.

Enomé, S. 1971, Report UAG-12, WDC-A, Part I, p. 88.

Evans, L. G., Fainberg, J., and Stone, R. G. $\quad$ 1971, Solar Phys. 21, 198.

Eviatar, A., and Dryer, M. 1970, Cosmic Electrodynamics 1, 371. 
Eyles, C. J., Linney, A. D., and Rochester, G. K. 1972, Solar Phys. 24, 483.

Fainberg, J., and Stone, R. G. 1970a, Solar Phys. 15, 222.

Fainberg, J., and Stone, R. G. $\quad$ 1970b, Solar Phys. 15, 433.

Fainberg, J., and Stone, R. G. $\quad$ 1971, Solar Phys. 17, 392.

Falciani, A., and Rigutti, M. 1972, Solar Phys. 26, 114.

Fay, T. D., Wyller, A. A., and Yun, H. S. $\quad$ 1972, Solar Phys. 23, 58.

Fehlau, P. E., Chambers, W. H., Fuller, J. C., Kunz, W. E., Milkey, R. W., and Blocker, N. K. 1971, Nature 232, 42.

Fehlau, P. E. $\quad$ 1971, Solar Phys. 20, 228.

Fisher, R. 1971, Solar Phys. 19, 440.

Fisk, L. A. $\quad 1971$, J. Geophys. Res. 76, 1662.

Fisk, L. A., and Schatten, K. H. 1972, Solar Phys. 23, 204.

Fleischer, R. L., Hart, H. R., and Comstock, C. M. 1970, G. E. Report No. $71-C$-021.

Fokker, A. D. 1970, Solar Phys. 11, 92.

Fokker, A. D. 1971, Proc. Second Meeting of CESRA, Trieste, p. 110.

Formisano, V., Palmiotto, F., and Moreno, G. 1970, Solar Phys. 15, 479.

Fortini, T., and Torelli, M. 1970, Solar Phys. 11, 425.

Foukal, B. 1971a, Solar Phys. 19, 59.

Foukal, B. $\quad$ 1971b, Solar Phys. 20, 298.

Frazier, E. N. 1971, Solar Phys. 21, 42.

Frazier, E. N. 1972a, Solar Phys. 24, 98.

Frazier, E. N. 1972b, Solar Phys. 26, No. 1.

Frazier, E. N., and Stenflo, J. O. $\quad$ 1972, Solar Phys. 27, 330.

Fredga, K. 1971, Solar Phys. 21, 60.

Freeman, F. F., and Jones, B. B. 1970, Solar Phys. 15, 288.

Freeman, F. F., Gabriel, A. H., Jones, B. B., and Jordan, C. $\quad$ 1971, Phil. Trans. Roy. Soc.-A 270, 127.

Friis-Christensen, E. $\quad$ 1971, Danish Meteorol. Inst. Geophys. Papers R-27.

Friis-Christensen, E., Lassen, K., Wilcox, J., Gonzalez, W., and Colburn, D. $\quad$ 1971, Nature Phys. Sci. 233, 48.

Friis-Christensen, E., Lassen, K., Wilhjelm, J., Wilcox, J., Gonzalez, J., and Colburn, D. $\quad$ 1972, J. Geophys. Res. 77, 3371.

Fritzova, L., and Švestka, Z. $\quad$ 1971, Solar Phys. 17, 212.

Frost, K. J. $\quad 1969$, Astrophys. J. 158, L159.

Frost, K. J., and Dennis, B. R. 1971, Astrophys. J. 165, 655.

Gabriel, A. H. 1971, Solar Phys. 21, 392.

Gabriel, A. H. $\quad 1972$, Mon. Not. R. astr. Soc., to be published.

Gabriel, A. H., and Jordan, C. 1969a, Nature 221, 947.

Gabriel, A. H., and Jordan, C. 1969 b, Mon. Not. R. astr. Soc. 145, 241.

Gabriel, A. H., and Jordan, C. $\quad 1970$, Physics Letters 32A, 166.

Gabriel, A. H., and Jordan, C. 1972, Astrophys. J., to be published.

Gabriel, A. H., Garton, W. R. S., Goldberg, L., Jones, T. J. L., Jordan, C., Morgan, F. J., Nicholls, R. W., Parkinson, W. J., Paxton, H. J. B., Reeves, E. M., Shenton, C. B., Speer, R. J., and Wilson, R. 1971, Astrophys. J. 169, 595.

Gabriel, A. H., and Paget, T. M. 1972, Journ. of Phys.-B, to be published.

Garcia, C., Hansen, R., Hull, H., Lacey, L., and Lee, R. 1971, Bull. A. A. S. 3, 261.

Garczynska, I., and Rompolt, B. 1971, '6th Regional Consultation on Solar Physics', Gyula, Hungary.

Geiss, J., Eberhardt, P., Buhler, F., and Meister, J. 1970, J. Geophys. Res. 75, 5972.

Gel'freikh, G. B., Zhitnik, I. A., and Livshits, M. A. 1970, Astron. Zh. 47, 329.

Gibson, J., and Van Allen, J. A. 1970, Astrophys. J. 161, 1135.

Giovanelli, R. G. 1971, IAU Symp. 43, 293.

Giovanelli, R. G. $\quad$ 1972, Solar Phys. 27, 71.

Gleissberg, W. 1971, Mitt. der Astron. Gesett. 30, 151.

Glencross, W. M. 1971, Phil. Trans. Roy. Soc. Lond. A 270, 117.

Gokhale, M. H., and Zwaan, C. 1972, Solar Phys. $26,52$.

Goldsmith, D. 1971, Solar Phys. 19, 86.

Godoli, G., Motta, S., and Zappala, A. 1971, Boll. SIF 87, 49.

Gopasyuk, S. I., and Tsap, T. T. 1971, IAU Symp. 43, p. 274.

Gosling, J. T. 1971, Solar Phys. 17, 499. 
Gosling, J. T., Hansen, R. T., and Bame, S. J. $\quad$ 1971, J. Geophys. Res. 76, 1811.

Gosling, J. T., and Bame, S. J. 1972, J. Geophys. Res. 77, 12.

Gosling, J. T., Pizzo, V., Neugebauer, M., Snyder, C. W. 1972a, J. Geophys. Res. 77, 2744.

Gosling, J. T., Hundhausen, A. J., Pizzo, V., and Ashbridge, J. R. 1972b, J. Geophys. Res. $77,5442$.

Graedel, T. E. 1970 , Astrophys. J. 160, 301.

Greenstadt, E. W., Green, I. M., Inouye, G. T., and Sonett, C. P. $\quad$ 1970, Planet. Space Sci. $18,333$.

Grevesse, N., and Swings, J. P. $\quad$ 1971, Astron. Astrophys. 13, 329.

Grigorev, V. M., and Kuklin, G. V. 1971, IAU Symp. 43, p. 252.

Grigorjev, V. M., and Katz, J. M. 1972, Solar Phys. 22, 119.

Grineva, Yu. I., Karev, V. I., Korneev, V. V., Krutov, V. V., Mandelshtam, S. L., Vainshtein, L. A., Vasiljev, B. N., and Zhitnik, I. A. 1972, Kosmicheskie Issledovaniya, in press.

Grossi Gallegos, H., Molnar, M., and Seibold, J. R. 1971, Solar Phys. 16, 120.

Gupta, M. K., and Sarkar, S. K. 1971, Indian J. of Pure and Applied Phys. 9, 249.

Gurtovenko, E. A. $\quad$ 1967, Voporsy Astrofiziky, Kiev, p. 74.

Guseynov, M. D. $\quad$ 1970, Izv. Krimskoj Astrofiz. Obs. 41-42, 89.

Haddock, F. T., and Graedel, T. E. 1970, Astrophys. J. 160, 293.

Hagen, J. P., and Neidig, D. F. 1971, Solar Phys. 18, 305.

Hakura, Y., Nishizaki, R., Tao, K., and Yamashita, F. $\quad$ 1970, J. Radio Res. Labs. Japan $17,21$.

Hall, D. N. B., Noyes, R. W., and Ayres, T. R. 1972, Astrophys. J. 171, 615.

Hall, D. N. B., and Noyes, R. W. 1972, Astrophys. J. 175, L95.

Hall, L. A. 1971, Solar Phys. 21, 167.

Hall, L. A., and Hinteregger, H. E. 1969, in Solar Flares and Space Research, de Jager and Švestka (eds.), p. 81 (Reidel, Dordrecht).

Hall, L. A., and Hinteregger, H. E. $\quad 1970$, J. Geophys. Res. 75, 6959.

Hansen, R. T., Garcia, C. J., Hansen, S. F., and Loomis, H. G. 1969 , Solar Phys. 7, 417.

Hansen, R. T., Garcia, C. J., Grognard, R. J.-M., and Sheridan, K. V. 1971 , Proc. A. S. A. $2,57$.

Hansen, R. T., Hansen, S. F., and Garcia, C. 1972, Proceedings of the Conference on Flare-Produced Shock Waves in the Corona. National Center for Atmospheric Research, Boulder, 11-14 Sept. 1972, in press.

Harries, J. R. 1970, Solar Phys. 13, 467.

Harvey, J. W. 1971, Publ. Astron. Soc. Pac. 83, 539.

Harvey, J. W. 1972, Solar Phys. 24, 354.

Harvey, K. L., Livingston, W. C., Harvey, J. W., and Slaughter, C. D. $\quad$ 1971, IAU Symp. 43, p. 422.

Harvey, K. L., and Martin, S. F. $\quad$ 1972, Lockheed Rep. LMSC D 269923, p. 24.

Hashim, A., and Bercovitch, M. 1972, Planet. Space Sci. 20, 791.

Haug, E. 1972, Solar Phys. 25, 425.

Hauge, O.. 1971, Astron. Astrophys. 10, 73.

Haurwitz, M. W. 1972, submitted to Planet. Space Sci.

Havnes, O. 1970, Solar Phys. 13, 323.

Heppner, J. 1972, Symp. on Critical Problems of Magnetospheric Phys., COSPAR, Madrid, May 1972, Goddard Sp. Flt. Ctr. Rept. X-645-77-154.

Heristchi, Dj., and Trottet, G. 1971, Phys. Rev. Letters 26, 197.

Heristchi, Dj., LeGrand, J. P., and Petrou, D. 1971 , Solar Phys. 18, 321.

Herring, J. R. H., and Craig, I. J. D. 1973, Solar Phys., 28, 169.

Heroux, L., Cohen, M., and Malinovsky, M. 1972, Solar Phys. 23, 369.

Hildner, E. 1971, Ph.D. Thesis, Univ. of Colorado.

Hinteregger, H. E., and Hall, L. A. 1969, Solar Phys. 6, 175.

Hirayama, T. 1971a, Solar Phys. 17, 50.

Hirayama, T. 1971 b, Solar Phys. 19, 384.

Hirayama, T. 1972, Solar Phys., 24, 310.

Hirshberg, J., Alksne, A., Colburn, D. S., Bame, S. J., Hundhausen, A. J. 1970, J. Geophys. Res. $75,1$. Hirshberg, J., Asbridge, J. R., and Robbins, D. E. 1971 , Solar Phys. 18, 313.

Hirshberg, J., Bame, S. J., and Robbins, D. E. 1972a, Solar Phys. 23, 467.

Hirshberg, J., Asbridge, J. R., and Robbins, D. E. 1972b, J. Geophys. Res. 77, 3583.

Hollweg, J. V. 1970, J. Geophys. Res. 75, 3715.

Holt, S. S., and Ramaty, R. 1969, Solar Phys. 8, 119.

Holzer, T. E., and Axford, W. I. 1970, J. Geophys. Res. 75, 6354.

Horan, D. M. 1971, Solar Phys. 21, 188. 
Houminer, Z. 1971, Nature Phys. Sci. 231, 165.

Houminer, Z., and Hewish, A. $\quad$ 1972, Planet. Space Sci. 20, 1703.

Howard, R. (ed.) 1971, Solar Magnetic Fields, IAU Symp. 43.

Howard, R., and Stenflo, J. O. 1972, Solar Phys. 22, 402.

Howard, R. 1972a, Solar Phys. 24, 123.

Howard, R. 1972b, Solar Phys. 25, 5.

Hsieh, K. C., and Simpson, J. A. 1970, Astrophys. J. 162, L191.

Hudson, H. S. 1972, Solar Phys. 24, 414.

Hudson, H. S., and Ohki, K. 1972, Solar Phys. 23, 155.

Hundhausen, A. J. 1970a, Rev. of Geophys. 8, 729.

Hundhausen, A. J. 1970b, Intercorrelated Satellite Observations Related to Solar Events, V. Manno and D. E. Page (eds.), p. 111 (Reidel, Dordrecht).

Hundhausen, A. J. 1972, Coronal Expansion and Solar Wind (Springer-Verlag, N. Y.).

Hundhausen, A. J., Bame, S. J., and Montgomery, M. D. 1971, J. Geophys. Res. 76, 5145.

Ito, K., and Okazoe, H. $\quad$ 1970, Acta Physica Academiaca Scientrum Hungaricae 29, 679.

Ivanov, K. G., and Mikerina, N. V. 1970, Geomagnetism and Aeronomy 10, 1075.

Jäger, F. W. 1972, Solar Phys. 27, 481.

Jakimiec, J. 1970, Astron. Zh. Akad. Nauk SSSR 47, 520.

Jakimiec, J. 1971, IAU Symp. 43, p. 505.

Janssens, T. J. $\quad 1972$ a, Bull. $A$. A. S. 4, 385.

Janssens, T. J. $\quad 1972 \mathrm{~b}$, Solar Phys. 25, 237.

Janssens, T. J., and White, K. P., III. 1970, Solar Phys. 11, 299.

Jensen, E. 1972, Ana Capri Colloq. on The Physics of Solar Prominences, Solar Phys. 24, 3.

Jokipii, J. R. 1970, Astrophys. J. 161, 1147.

Jokipii, J. R., and Hollweg, J. V. 1970, Astrophys. J. 160, 745.

Jokipii, J. R., and Lee, L. C. $\quad$ 1972, Astrophys. J. 172, 729.

Jones, T. B. 1971, J. Atmos. Terr. Phys. 33, 963.

Jones, T. L. J., Parkinson, W. H., Speer, R. J., and Yang, C. $\quad$ 1971, Solar Phys. 21, 372.

Jordan, C. 1971, Solar Phys. 21, 381.

Jorgensen, T., Friis-Christensen, E., and Wilhjelm, J. $\quad$ 1972, J. Geophys. Res. 77, 1976.

Kahler, S. W. $\quad 1971$, Astrophys. J. 164, 365.

Kahler, S. W. 1972, Solar Phys. 25, 435.

Kahler, S. W., Meekins, J. F., Kreplin, R. W., and Bowyer, C. S. $\quad$ 1970, Astrophys. J. 162, 293.

Kahler, S. W., and Kreplin, R. W. 1970, Solar Phys. 14, 372.

Kahler, S. W., and Kreplin, R. W. 1971 , Astrophys. J. 168, 531.

Kai, K. 1970, Solar Phys. 11, 310.

Kakinuma, T., Yamashita, T., and Enome, S. $\quad$ 1969, Proc. Res. Inst. Atmospherics 16, 127.

Kaminer, N. S., Kashevarov, V. P., Kuzmicheva, A. E., and Dorman, L. I. 1972, Geomag. $i$ Aetron. $12,385$.

Kane, S. R. $\quad 1969$, Astrophys. J. 157, L139.

Kane, S. R. $\quad 1971$, Astrophys. J. 170, 587.

Kane, S. R. $\quad$ 1972, Solar Phys. 27, 174.

Kane, S. R., and Winckler, J. R. 1969, Solar Phys. 6, 151.

Kane, S. R., and Anderson, K. A. $\quad 1970$, Astrophys. J. 162, 1003.

Kane, S. R., and Hudson, H. S. $\quad$ 1970, Solar Phys. 14, 414.

Kane, S. R., and Donnelly, R. F. 1971, Astrophys. J. 164, 151.

Kane, S. R., and Lin, R. P. $\quad 1972$, Solar Phys. 23, 457.

Kane, S. R., Kahler, S. W., and Kurfess, J. D. 1972, Solar Phys. 25, 418.

Karaev, A. A. $\quad 1970$, Akad. Nauk. USSR Soln. Dann. 12, 107.

Kaufmann, P., and Mendes, A. 1970, J. Atmos. and Terr. Phys. 32, 427, C.R.A.A., Mackenzie Univ. Sao Paulo, Brazil.

Kavanagh, L. D., Jr., Schardt, A. W., and Roelof, E. C. $\quad$ 1970, Rev. Geophys. 8, 389.

Kawasaki, K., Yasuhara, F., and Akasofu, S.-I. 1972, Trans. Am. Geophys. Union 53, 503.

Keath, E. P., Bukata, R. P., McCracken, K. G., and Rao, U. R. $\quad$ 1971, Solar Phys. 18, 503.

Kelly, P. T., and Rense, W. A. $\quad 1972$, Solar Phys. 26, 431.

Kimpara, A., and Nishiwaki, A. $\quad$ 1971, Comptes Rendus del'Acad. Francaise de Paris, Ser. B 272, 1493.

Kinsey, J. H. 1970, Phys. Rev. Lett. 24, 246.

Kirsch, E., and Munch, J. W. $\quad 1972$, Planetary and Space Sci. 20, 89. 
Kirsch, E. 1973, Solar Phys. 28, 233.

Kirshner, R. P., and Noyes, R. W. 1971, Solar Phys. 20, 428.

Kissel, K. E., Morais, C., Righini, A., and Righini, G. $\quad$ 1970, Appl. Opt. 9, 2620.

Kleczek, J., Leroy, J.-L., and Orrall, F. Q. 1972, 'A General Bibliography of Solar Prominence Research 1880-1970', Publ. Astr. Inst. Czech. 53.

Kneer, F. $\quad 1972 a$, Astron. Astrophys. 18, 39.

Kneer, F. $\quad 1972$ b, Astron. Astrophys. 18, 47.

Kocharov, G. E., Charikov, Iu. E., Karchenko, A. A., Gusev, G. V., and Baskakov, A. V. $\quad$ 1972, Letters v ZhETF 15, 153.

Kodama, M., Kusunose, M., and Ogura, K. 1971, Rept. of Ionosp. and Space Res. in Japan 25, 285.

Kondratjev, K. Y., and Nikolsky, G. A. 1970, Quart. J. Roy. Met. Soc. 96, 509.

Konyakhina, S. S., Kurnosova, L. V., Logachev, V. I., Razorenov, L. A., and Fradkin, M. I. 1972, P. N. Lebedev Physical Institute, Preprint No. 74.

Koomen, M. J., Purcell, J. D., and Tousey, R. 1970, Nature 226, 1135.

Kopp, R. A. $\quad 1972$, Solar Phys. $27,373$.

Korchak, A. A. 1971, Solar Phys. 18, 284.

Korchak, A. A. 1972, Inst. of Terr. Magnetism, Ionosphere and Radiowave Propagation, USSR, Preprint No. 9.

Kordylewski, Z., Mergentaler, J., Jakimiec, J., Sylwester, B., and Sylwester, J. $\quad$ 1972, COSPAR W.G. 3, Panel 3B, Paper d.15.

Kostik, R. I. 1971a, Akad. Nauk. USSR, Soln. Dan. 2, 80.

Kostik, R. I. $\quad$ 1971b, Astr. Zhur. 48, 738.

Kotov, V. A. $\quad 1970$, Izv. Krimskoj Astrofiz. Obs. 41-42, 67.

Kotov, V. A. 1971, IAU Symp. 43, 212.

Kotsarenko, N. Ya., Koshevaya, S. V., and Yukhimuk, A. K. 1970, Geomagnetism and Aeronomy 10, 715.

Kovalevsky, J. V. $\quad$ 1971, Space Sci. Rev. 12, 187.

Krat, V. A., Karpinsky, V. N., and Pravdjuk, L. M.

Krause, F., and Rädler, K.-H. 1971 , IAU Symp. 43, p. 770.

Kreplin, R. W., and Taylor, R. G. $\quad$ 1972, Solar Phys. 21, 452.

Krieger, A. S., Vaiana, G. S., and Van Speybroeck, L. P. 1971 , IAU Syrmp. 43, p. 397.

Krieger, A., Paolini, F., Vaiana, G. S., and Webb, D. $\quad$ 1972, Solar Phys. 22, 150.

Krimigis, S. M., and Verzariu, P. 1971, J. Geophys. Res. 76, 792.

Krivský, L. 1970, Bull. Astron. Inst. Czech. $21,67$.

Krivský, L., Tlamicha, A., Halenka, J., Lastovicka, J., Tríska, P., Pintér, S., and llencik, J. $\quad$ 1972, Bull. Astron. Inst. Czech. 23, 94.

Kubota, J., and Leroy, J.-L. 1970, Astron. Astrophys. 6, 275.

Kubotz, J., Tamenaga, T., and Yoshikawa, K. 1972, Publ. Astr. Soc. Japan 24, 343.

Kuiper, T. B. H., and Pasachoff, J. M. 1973, Solar Phys. 28, 187.

Künzel, H. 1971, Astron. Nachr. 293, 105.

Kupetsky, V. N. 1971, Soln. Dan. 3, 107.

Kurochka, L. N. $\quad 1969$, Astron. Zh. 46, 85.

Kurochka, L. N. $\quad 1970$, Astron. Zh. 47, 111.

Kurochka, L. N., and Maslennikova, L. B. $\quad$ 1970, Solar Phys. 11, 33.

Kuzmicheva, A. E., Dorman, L. I., and Kaminer, N. S. $\quad$ 1972, Geomag. $i$ Aeron. 12, 534.

Labrum, N. R., and Stewart, R. T. $\quad 1970$, Proc. Astron. Soc. Australia 1, 316.

Lal, D., Lorin, J. C., Pellas, P., Rajan, R. S., and Iamhane, A. S. 1972, Meteorite Research, Millman, ed.

Lambert, D. L., and Mallia, E. A. 1971 , Mon. Not. R. astr. Soc. 151, 437.

Lambert, D. L., Mallia, E. A., and Petford, A. D. 1971, Mon. Not. R. astr. Soc. 154, 265.

Lambert, D. L., and Mallia, E. A. $\quad 1972$, Mon. Not. R. astr. Soc. 156, 337.

Landini, M., and Monsignori Fossi, B. C. 1970, Astron. Astrophys. 6, 468.

Landini, M., Monsignori Fossi, B. C., Poletto, G., Russo, D., and Tagliaferri, G. L. $\quad$ 1970, Solar Phys. $13,226$.

Landini, M., and Monsignori Fossi, B. C. 1971, Solar Phys. 17, 379.

Landini, M., Monsignori Fossi, B. C., and Pallavicini, R. 1973, Solar Phys. 29, 93.

Lange, J., and Scherb, F. 1970, J. Geophys. Res. 75, 6350.

Lanzerotti, L. J. $\quad 1969$, Report UAG-5, WDC-A, p. 56.

Lanzerotti, L. J., and Robbins, M. F. 1969, Solar Phys. 10, 212. 
Lanzerotti, L. J., and Maclennan, C. G. 1971, Paper presented at the COSPAR Symp. on November 1969. Solar Particle Event, Boston.

Lazarus, A. J., Ogilvie, K. W., and Burlaga, L. F. $\quad$ 1970, Solar Phys. 13, 232.

Lehnert, B. 1971 Nuclear Fusion 11, 485.

Leroy, J.-L., Muller, R., and Poulain, P. 1972, Astron. Astrophys. 17, 301.

Levitskii, L., and Vladimirskii, V. $\quad 1969$, Izv. Krimsk. Astrophys. Ob. 161-172.

Levy, G. S., Sato, T., Seidel, B. L., Stelzried, C. T., Ohlson, J. E., and Rusch, W. V. T. 1969, Science $166,596$.

Lin, R. P. 1970a, Solar Phys. 12, 209.

Lin, R. P. 1970b, Solar Phys. 12, 266.

Lin, R. P. 1970c, Solar Phys. 15, 453.

Lin, R. P. 1971, Proc. of the XII International Cosmic Ray Conference, Hobart 5, 1805.

Lin, R. P. 1972, Paper presented at the Solar Terrestrial Relation Conference, Calgary.

Lin, R. P., Kahler, S. W., and Roelof, E. C. $\quad$ 1969, Solar Phys. 4, 338.

Lin, R. P., and Hudson, H. S. 1971, Solar Phys. 17, 412.

Lin, R. P., Anderson, K. A., Cline, T. L. 1972, Phys. Rev. Lett., in press.

Lockwood, J., Lezniak, J., and Webber, W. 1972, J. Geophys. Res. 77, 4839.

Loginov, V. F., and Sukhomazova, G. I. 1970, Soln. Dan. 5, 109.

Loginov, V. F., and Sukhomazova, G. I. 1971, Geomagnetism and Aeronomy 11, 335.

Lovelace, R. V. E., Salpeter, E. E., Sharp, L. E., and Harris, D. E. 1970, Astrophys. J. 159, 1047. Machado, M. E. $\quad 1971$, Solar Phys. 17, 389.

Machado, M. E., and Seibold, J. R. 1973, Solar Phys. 29, 75.

Mallia, E. A., Blackwell, D. E., and Petford, A. D. 1971 , Solar Phys. 20, 369.

Mallia, E. A., and Petford, A. D. $\quad$ 1972, Mon. Not. R. astr. Soc. 157, 73.

Maltby, P. $\quad$ 1970, Solar Phys. 13, 312.

Maltby, P. 1971a, IAU Symp. 43, p. 141.

Maltby, P. 1971b, Solar Phys. 18, 3.

Maltby, P. 1972, Solar Phys. 26, 76.

Maltby, P., and Staveland, L. 1971, Solar Phys. 18, 443.

Malville, J. M., and Tandberg-Hanssen, E. $\quad 1973$, Bull. A. A. S., in press.

Mamadazimov, M. 1972, Solar Phys. 22, 129.

Mandelshtam, S. L. $\quad$ 1971, Phil. Trans. Roy. Soc. Lond.-A 270, 135.

Manno, V., and Page, D. E. $\quad$ 1970, Intercorrelated Satellite Observations Related to Solar Events (Reidel, Dordrecht).

Martin, S. 1972, Ana Capri Colloq. on The Physics of Solar Flares, Solar Phys. $24,3$.

Martres, M. J., Soru-Escaut, I., and Rayrole, J. 1971, IAU Symp. 43, p. 435.

Martres, M. J., and Soru-Escaut, I. 1972, Solar Phys. 21, 137.

Matheson, D. N., and Little, L. T. $\quad$ 1971, Planet. Space Sci. 19, 1615.

Mattig, W. 1971, Solar Phys. 18, 434.

Maxwell, A. 1971, Solar Phys. 16, 224.

Maxwell, A., and Fitzwilliam, J. 1972, Astrophys. Letters, in press.

Mayfield, E. B. $\quad 1971$, IAU Symp. 43, p. 376.

McAllister, H. C. 1971, Solar Phys. 21, 27.

McCabe, M. K. $\quad$ 1970, Solar Phys. 12, 115.

McCabe, M. K. 1971, Solar Phys. 19, 451.

McCabe, M. K., and Fisher, R. R. 1970, Solar Phys. 14, 212.

McLean, D. J. $\quad$ 1970, Proc. Astron. Soc. Australia 1, 315.

McCracken K. G., and Rao, U. R. 1970, Space Rev. 11, 155.

McCracken, K. G., Rao, U. R., Bukata, P. R., and Keath, E. P. $\quad$ 1971, Solar Phys. 18, 100.

McDonald, F. B., and Desai, U. D. 1971, J. Geophys. Res. 76, 808.

McDonald, F. B., Van Hollebeke, M., and Wang, J. 1972, Paper presented at American Astro. Soc., Solar Physics Div. Meeting, Apr. 4-6, Univ. of Maryland.

McIntosh, P. S., and Donnelly, R. F. 1972, Solar Phys. 23, 444.

McKenna-Lawlor, S. M. P. $\quad$ 1970, Astrophys. J. 159, 51.

McKenzie, D. L. 1972 , Astrophys. J. 175, 481.

McKenzie, D. L., Datlowe, D. W., and Peterson, L. E. $\quad$ 1973, Solar Phys. 28, 175.

McKibben, R. B. 1972, J. Geophys. Res. 77, 3957.

Meekins, J. F., and Doschek, G. A. 1970, Solar Phys. 13, 213. 
Meekins, J. F., Doschek, G. A., Friedman, H., Chubb, T. A., and Kreplin, R. W. 1970, Solar Phys. 13, 198.

Melrose, D. B. $\quad 1970$, Australian J. Phys. 23, 885.

Mewe, R. 1972a, Solar Phys. 22, 114.

Mewe, R. $\quad 1972 b$, Solar Phys. 22, 459.

Meyer, R. X., and Mayfield, E. B. $\quad$ 1972, Bull. A. A. S. 4, 387.

Michard, R. $\quad 1971$, IAU Symp. 43, 359.

Mickey, D. L. 1972, submitted to Astrophys. J. (Letters).

Mickey, D. L., and Orrall, F. Q. 1972, submitted to Astron. Astrophys.

Milkey, R. W. 1971 , Solar Phys. 16, 465.

Milkey, R. W., Blocker, N. K., Chambers, W. H., Fehlau, P. E., Fuller, J. C., and Kunz, W. E. 1971, Solar Phys. 20, 400.

Miller, R. A. 1971 , Solar Phys. 16, 373.

Milovidova, N. P., and Nefedév, V. P. 1971, Soln. Dan. 7, 109.

Mitra, S. N. $\quad$ 1970, Solar Phys. 15, 249.

Mogro-Campero, A., and Simpson, J. A. $\quad$ 1972, Astrophys. J. 171, 15.

Molnar, H. 1972, Astron. Astrophys. 20, 69.

Montgomery, M. D., Bame, S. J., and Hundhausen, A. J. $\quad$ 1972, J. Geophys. Res. 77, 5432.

Morozhenko, N. N. $\quad 1970$, Akad. Nauk. USSR Soln. Dan. $12,89$.

Morozhenko, N. N. 1971a, Akad. Nauk. USSR, Soln. Dan. 6, 67.

Morozhenko, N. N. $\quad 1971$ b, Astr. Zhur. 48, 1237.

Morozhenko, N. N. $\quad 1971$ c, Akad. Nauk. USSR, Soln. Dan. 8, 81.

Munro, R. H., Dupree, A. K., and Withbroe, G. L. $\quad 1971$, Solar Phys. 19, 347.

Mykland, N. $\quad 1973$, Solar Phys., 28, 49.

Najita, K., and Orrall, F. Q. $\quad$ 1970, Solar Phys. 15, 176.

Nakagawa, Y. $\quad$ 1970, Solar Phys. 12, 419.

Nakagawa, Y. 1971, IAU Symp. 43, p. 725.

Nakagawa, Y., Raduu, M. A., Billings, D. E., and McNamara, D. 1971 , Solar Phys. 19, 72.

Nakagawa, Y., Raduu, M. A., and Harvey, J. W. 1972, Solar Phys., in press.

Neher, H. V. 1971, J. Geophys. Res. 76, 1637.

Ness, N. F., Hundhausen, A. J., and Bame, S. J. 1971, J. Geophys. Res. 76, 6643.

Nesterov, G. $\quad$ 1971, J. Atmos. Terr. Phys. 33, 119.

Neupert, W. M. 1971a, Solar Phys. 18, 474.

Neupert, W. M. 1971b, Phil. Trans. Roy. Soc. Lond.-A 270, 143.

Neupert, W. M., and Swartz, M. 1970, Astrophys. J. 160, L189.

Newkirk, G. N., Jr. $\quad 1972$, Asilomar Conference on Solar Wind.

Newkirk, G., Jr., and Altschuler, M. D. 1970 , Solar Phys. 13, 131.

Newkirk, G., Jr., Trotter, D. E., Altschuler, M. D., and Howard, R. 1972 , Solar Phys., in press.

Noyes, R. W. 1971, Ann. Rev. Astron. Astrophys. 9, 209.

Noyes, R. W., Withbroe, G. L., and Kirshner, R. P. $\quad$ 1970, Solar Phys. 11, 388.

Noyes, R. W., and Kalkofen, W. 1970, Solar Phys. 15, 120.

Noyes, R. W., Dupree, A. K., Huber, M. C. E., Parkinson, W. H., Reeves, E. M., and Withbroe, G. L.

1972, Solar Satellite Project, Harvard College Observatory, June $1972 \mathrm{Tr}-35$.

Obridko, V. N., and Demkina, L. B. 1972, Solar Phys. 24, 336.

Ogilvie, K. W., and Arens, J. F. $\quad$ 1971, J. Geophys. Res. 76, 13.

Ohki, K. 1969, Solar Phys, 7, 260.

Öhman, Y. 1971a, Trans. I.A.U. XIV. B, p. 105.

Ohman, Y. $\quad$ 1971b, Solar Phys. 24, 3.

Öhman, Y. 1972a, Solar Phys. 23, 134.

Öhman, Y. $\quad 1972 b$, Nobel Symp. 21, 343.

Ohshio, M. 1971, Nature Phys. Sci. 229, 239.

Oksman, J. 1971, Nature Phys. Sci. 231, 12.

Olsen, J. 1948, Terr. Magn. and Atmos. Elec. 53, 123.

Orrall, F. Q. 1971, Solar Phys. 24, 3.

Orrall, F. Q., and Smith, H. J. 1961, Sky and Tel. 22, 330.

Oster, L., Mariska, J., Altschuler, M., and Trotter, D. 1972. Bull. A. A. S. 4, 389.

Palmer, I. D., and Smerd, S. F. 1972, Solar Phys. 26, 460.

Parkinson, J. H. 1971, Nature Phys. Sci. 233, 44. 
Parkinson, J. H. $\quad$ 1972, Nature Phys. Sci. 236, 68.

Parkinson, J. H., and Pounds, K. A. 1971, Solar Phys. 17, 146.

Parks, G. K., and Winckler, J. R. 1971, Solar Phys. 16, 186.

Pathak, P. N. 1971, J. Geophys. Res. 76, 7804.

Pathak, P. N., and Sarabhai, V. 1970 , Planet. and Space Sci. 18, 81.

Phillis, G. L., and Ramsey, H. E. $\quad$ 1972, Lockheed Rept. LMSC D 269923, p. 1.

Phillips, K. J. H., and Culhane, J. L. 1971, Solar Phys. 16, 469.

Piddington, J. H. 1971a, Solar Phys. 21, 4.

Piddington, J. H. 1971b, Proc. Astron. Soc. Australia 2, 7.

Piddington, J. H. 1972, Solar Phys. 22, 3.

Pikel'ner, S. B. 1971a, Solar Phys. 17, 44.

Pikel'ner, S. B. 1971b, Solar Phys. 20, 286.

Pinter, S. 1969, Academia, nakladatelstvi Ceskoslovenske akademie ved. 73.

Pinter, S. 1969, Solar Phys. 8, 149.

Pneuman, G. W. 1972, Astrophys. J.

Poland, A., Skumanich, A., Athay, R. G., and Tandberg-Hanssen, E. $\quad$ 1971, Solar Phys. 18, 391.

Poland, A., and Anzer, U. 1971, Solar Phys. 19, 401.

Prata, S. W. 1971, Solar Phys. 20, 310.

Price, P. B., and Sullivan, J. D. $\quad 1971$, 12th International Conference on Cosmic Rays 2, 449.

Price, P. B., Hutcheon, I., Cowsik, R., and Barber, D. J. $\quad 1971$, Phys. Rev. Lett. $26,916$.

Purcell, J. D., and Tousey, R. 1970, Solar-Terrestrial Phys., E. R. Dyer, ed. (Reidel, Dordrecht).

Purcell, J. D., and Widing, K. G. $\quad$ 1972, Astrophys. J. 178, 239.

Raadu, M. A., and Nakagawa, Y. 1971, Solar Phys. $20,64$.

Raadu, M. A., and Kuperus, M. 1972, in preparation.

Rakhubovsky, A. S. 1972, Astrometr. and Aph. 17.

Razmadze, T. S., and Berishvili, G. P. 1971, Soln. Dan. 2, 111.

Rao, U. R., McCracken, K. G., Allum, F. R., Palmeira, R. A. R., and Bartley, W. C. 1971, Solar Phys. 19, 209.

Rayrole, J. 1971, IAU Symp. 43, p. 181.

Rayrole, J., and Semel, M. 1970, Astron. Astrophys. 6, 288.

Reeves, E. M., and Parkinson, W. H. 1970, Astrophys. J. Suppl. $21,1$.

Reeves, E. M., and Parkinson, W. H. 1972, Solar Phys. 24, 113.

Ribes, E. $\quad 1969$, Astron. Astrophys. 2, 316.

Riddle, A. C. $\quad 1970$, Solar Phys. 13, 448.

Robbins, D. E., Hundhausen, A. J., and Bame, S. J. $\quad$ 1970, J. Geophys. Res. 75, 1178.

Roberts, P. H., Jr. $\quad 1970$, Thesis, Cal. Inst. Tech. Pasadena. Roberts,

Roberts, W. O., and Olson, R. H. 1972a, Rev. Geophys. and Space Phys., to be published.

Roberts, W. O., and Olson, R. H. 1972b, J. Atmos. Sci., in press.

Rompolt, B. 1972, Solar Phys.

Rompolt, B., Smolkov, G. Y., Bashkirtzev, V. S., and Zubkova, G. N. 1971, Research in Geomagn. Aeronomy and Solar Phys. 20, 242.

Rosenberg, R., and Coleman, P., Jr. $\quad$ 1969, J. Geophys. Res. 74, 5611.

Rosenberg, R. L., Coleman, P. J., Jr., and Colburn, D. S. 1971, J. Geophys. Res. 76, 6661.

Rossbach, M., and Schröter, E. H. 1970 , Solar Phys. 12, 95.

Roy, J. R. 1972, Solar Phys. 26, 418.

Rugge, H. R., and Walker, A. B. C., Jr. $\quad$ 1970, Solar Phys. 15, 372.

Rugge, H. R., and Walker, A. B. C., Jr. $\quad$ 1971, Solar Phys. 18, 244.

Russell, C. T., and McPherron, R. L. 1972, submitted to J. Geophys. Res.

Rust, D. M. 1972, Solar Phys. 25, 141.

Rust, D. M., and Roy, J.-R. 1971 , IAU Symp. 43, p. 569.

Sakurai, K. $\quad$ 1971, Planet. Space Sci. 19, 1289.

Sakurai, K. $\quad$ 1971a, Solar Phys. 16, 125.

Sakurai, K. $\quad$ 1971b, Solar Phys. 16, 198.

Sakurai, K. 1971c, Solar Phys. 17, 459.

Sakurai, K. $\quad$ 1971d, Publ. Astron. Soc. Pac. 83, 23.

Sakurai, K. 1972a, Publ. Astron. Soc. Pac. 84, 531.

Sakurai, K. $\quad$ 1972, Solar Phys. 23, 142.

Sakurai, K. 1972c, Physics of Solar Cosmic Rays, to be published. 
Sakurai, K. $\quad$ 1972d, Publ. Astron. Soc. Pac. 84, in press.

Sawyer, C. $\quad 1971$, IAU Symp. 43, p. 316.

Sawyer, C., and Hansen, Shirley F. $\quad 1972$, Bull. A. A. S. 4, 390.

Sawyer, C., and Haurwitz, M. W. 1972, Solar Phys. 23, 429.

Scalise, E. $\quad$ 1970, Publ. Astron. Soc. Japan 22, 483.

Schadee, A. 1970, Solar Phys. 15, 345.

Schatten, K. H. 1970, Solar Phys. 12, 484.

Schatten, K. H. 1971, Rev. Geophys. 9, 773.

Schatten, K. H., and Wilcox, J. M. $\quad$ 1970, Acta. Phys. Acad. Sci. Hungaricae 29, Suppl. 2, 279.

Schatten, K. H., and Schatten, J. E. 1972, J. Geophys. Res. 77, 4858.

Scherrer, P. H., Wilcox, J. M., and Howard, R. 1972, Solar Phys. 22, 418.

Schleicher, H., and Schröter, E. H. 1971, Solar Phys. 17, 31.

Schmidt, H., Wagner, W., and Newkirk, G. 1972, Bull. A. A. S. 4, 390.

Scholer, M., Hovestadt, D., and Häusler, B. $\quad$ 1972, Solar Phys. 24, 475.

Schröter, E. H. $\quad 1971$, IAU Symp. 43, 167.

Schweizer, W., and Schmidtke, G. 1971, Astrophys. J. 169, L27.

Semel, M., and Soru-Escaut, I. $\quad$ 1971, Astron. Astrophys. 12, 340.

Sen, H. K., and White, M. L. $\quad$ 1972, Solar Phys. 23, 146.

Sengupta, P. R. 1971a, J. Atmos. Terr. Phys. 33, 1953.

Sengupta, P. R. 1971 b, Solar Phys. 17, 160.

Severny, A. 1971, IAU Symp. 43, p. 340.

Severny, A., Wilcox, J. M., Scherrer, P. H., Colburn, D. S. $\quad$ 1970, Solar Phys. 15, 3.

Shaw, M. L. $\quad$ 1972, Solar Phys. 27, 436.

Sheeley, N. R., Jr. 1971 a, IAU Symp. 43, p. 310.

Sheeley, N. R., Jr. 1971 b, Solar Phys. $20,19$.

Sheeley, N. R., Jr. $\quad$ 1972, Solar Phys. 25, 98.

Sheeley, N. R., Jr., and Engvold, O. 1970, Solar Phys. 12, 69.

Sheeley, N. R., Jr., and Bhatnagar, A. 1971, Solar Phys. 19, 338.

Sheridan, K. V. $\quad$ 1970, Proc. A. S. A. 1, 376.

Sheridan, K. V., Garcia, C. J., and Hansen, R. T. 1972, Bull. A. A. S. 4, 391.

Shimabukuro, F. I. $\quad 1970$, Solar Phys. 15, 424.

Shimabukuro, F. I. 1972, Solar Phys. 23, 169.

Shpitalnaja, A. A. $\quad$ 1971, Akad. Nauk. USSR Soln. Dann. 2, 99.

Shpitalnaja, A. A., and Vyalshin, G. F. 1970, Soln. Dann. 12, 74.

Simnett, G. M. 1971, Solar Phys. $20,448$.

Simnett, G. M. 1972a, Solar Phys. 22, 189.

Simnett, G. M. 1972b, Paper presented at the XIV Meeting of COSPAR, Madrid.

Simnett, G. M., and Holt, S. S. 1971, Solar Phys. 16, 208.

Simon, G. W., and Weiss, N. O. 1970, Solar Phys. 13, 85.

Singer, S., and Montgomery, M. D. 1971, J. Geophys. Res. 76, 6628.

Siscoe, G. L. 1972, J. Geophys. Res. 77, 27.

Smerd, S. F. $\quad$ 1967, Proc. Astron. Soc. Australia 1, 11.

Smerd, S. F. $\quad$ 1970, Proc. Astron. Soc. Australia 1, 305.

Smerd, S. F., and Dulk, G. A. 1971, IAU Symp. 43, p. 616.

Smith, D. F. $\quad$ 1970, Solar Phys. 15, 202.

Smith, S. F. $\quad$ 1971, IAU Symp. 43, p. 323.

Smith, D. F. $\quad$ 1972, Solar Phys. 23, 191.

Smith, S. F., and Harvey, K. L. 1971, 'Observational Effects of Flare-Associated Waves', preprint. Smith, D. F., and Pneuman, G. W. 1972, Solar Phys. 25, 461.

Smith, D. F., and Priest, E. R. 1972, Astrophys. J., in press.

Smolkov, G. Y., and Bashkirtsev, V. S. 1970, Soln. Dann. 12, 96.

Sonett, C. P., Coleman, P. J., Jr., Wilcox, J. M., eds. 1972, Solar Wind, Scientific and Technical Information Office, NASA, Washington, D. C.

Speer, R. J., Garton, W. R. S., Morgan, J. F., Nicholls, R. W., Goldberg, L., Parkinson, W. H., Reeves, E. M., Jones, T. J. L., Paxton, H. J. B., Shenton, D. B., and Wilson, R. 1970, Nature $226,249$. Staveland, L. $\quad$ 1970, Solar Phys. 12, 328.

Stein, A., and Zirin, H. 1972, Bull. A. A. S. 4, 392.

Stellmacher, G., and Wiehr, E. 1970, Astron. Astrophys. 7, 432. 
Stellmacher, G., and Wiehr, E.

1971a, Solar Phys. 17, 21.

Stellmacher, G., and Wiehr, E. $\quad$ 1971b, Solar Phys. 21, 96.

Stellmacher, G., and Wiehr, E. $\quad$ 1972, Astron. Astrophys. 19, 293.

Stenflo, J. O. 1970, Solar Phys. 14, 263.

Stenflo, J. O. 1972, Solar Phys. 23, 307.

Stenflo, J. O., and Frazier, E. N. 1972, Proc. Conf. on Line Formation in the Presence of Mag. Fields, Boulder, Colo.

Stepanyan, N. N. $\quad 1969$, Astron. Zh. 46, 580.

Stern, P. 1972, Goddard Sp. Flt. Ctr. Rept. X-641-72-133.

Steward, R. T., and Sheridan, K. V. 1970, Solar Phys. 12, 229.

Stoker, P. H., and Carmichael, Hugh 1971, Astrophys. J. 169, 357.

Stone, R. G., and Fainberg, J. 1972, Solar Phys. 20, 106.

Strauss, F. M., and Papagiannis, M. D. 1971, Astrophys. J. 164, 369

Sullivan, J. D. $\quad 1970$, Thesis, University of Chicago.

Svalgaard, L. $\quad$ 1972a, Bull. A. A. S. 4, 393.

Svalgaard, L. $\quad$ 1972b, J. Geophys. Res. 77, 4027.

Svestka, Z. 1970, Solar Phys. 13, 471.

Švestka, Z. 1971, Solar Phys. 19, 202.

Švestka, Z. 1972a, Ann. Rev. Astron. Astrophys. 10, 1.

Švestka, Z. 1972b, Solar Phys. 24, 154.

Sweet, P. A. $\quad$ 1969, Ann. Rev. Astron. Astrophys. 7, 149.

Syrovatsky, S. I. 1972a, Solar-Terrestrial Phys., Part I, de Jager, ed., p. 119 (Reidel, Dordrecht). Syrovatsky, S. I. 1972b, preprint.

Syrovatsky, S. I., and Shemleva, O. P. 1971, D. N. Lebedev Inst. Preprint No. 158

Takakura, T. 1969, Solar Phys. 6, 133.

Takakura, T. 1971, Solar Phys. 19, 186.

Takakura, T., and Scalise, E. $\quad$ 1970, Solar Phys. 11, 434.

Takakura, T., Ohki, K., Shibuya, N., Fujii, M., Matsuoka, M., Miyamoto, S., Nishimura, J., Oda, M., Ogawara, Y., and Ota, S. 1971, Solar Phys. 16, 454.

Takao, K., and Tsujii, T. 1970, Rept. of Ionosp. and Space Res. in Japan 24, 183

Tam, C. K. W., and Yousefian, V. 1972, J. Geophys. Res. 77, 234.

Tanaka, H., and Énomé, S. $\quad 1970$, Nature 225, 435.

Tanaka, H., and Énomé, S. $\quad$ 1971, Solar Phys. 17, 408.

Tanaka, K. 1972, Big Bear Report.

Tandberg-Hanssen, E. $\quad$ 1970, Solar Phys. 15, 359.

Tandberg-Hanssen, E. 1973, Solar Prominences (Gordon and Breach).

Tanenbaum, A. S., Wilcox, J. M., and Howard, R. 1971, IAU Symp. 43, p. 348.

Teegarden, B. J., von Rosenvinge, T. T., and McDonald, F. B. $\quad$ 1972, GSFC Preprint X-661-72-222, to be published in Astrophys. $J$.

Teske, R. G. 1971a, Solar Phys. 17, 76.

Teske, R. G. 1971b, Solar Phys. 17, 181.

Teske, R. G. 1971c, Solar Phys. 19, 356.

Teske, R. G. 1971d, Solar Phys. 21, 146.

Teske, R. G., and Thomas, R. J. 1969, Solar Phys. 8, 348.

Teske, R. G., Soyumer, T., and Hudson, H. S. 1971 , Astrophys. J. 165, 615.

Teske, R. G., and Thomas, R. J. 1972, Solar Phys. 24, 434.

Thomas, R. J., and Neupert, W. M. 1970, Bull. A. A. S. 3, No. 1, Part 1.

Thomas, R. J., and Teske, R. G. $\quad$ 1971, Solar Phys. 16, 431.

Thome, G. D., and Wagner, L. S. $\quad 1971$, J. Geophys. Res. 76, 6883.

Thomson, D. M. 1971, Planet. Space Sci. 19, 1169.

Timothy, A. F., and Timothy, J. G. $\quad$ 1970, J. Geophys. Res. 75, 6950.

Tindo, I. P., Ivanov, V. D., Mandelshtam, S. L., and Shuryghin, A. I. 1970, Solar Phys. 14, 204.

Tindo, I. P., Ivanov, V. D., Mandelshtam, S. L., Shuryghin, A. I., and Savel'ev, V. A. 1971, Kosmicheskie Issledovaniya 9, 116.

Tindo, I. P., Ivanov, V. D., Mandelshtam, S. L., and Shuryghin, A. I. 1972a, Solar Phys. $24,429$.

Tindo, I. P., Valnicek, B., Livshits, M. A., and Ivanov, V. D. $1972 b$, P. N. Lebedev Phys. Inst., Lab. of Spectroscopy Preprint No. 43.

Tindo, I. P., Ivanov, V. D., Mandelshtam, S. L., and Shuryghin, A. I. 1972c, Solar Phys. $24,429$. 
Title, A. M., and Andelin, J. P., Jr. $\quad$ 1971, IAU Symp. 43, p. 298.

Tomblin, F. F. $\quad$ 1972, Astrophys. J. 171, 377.

Tousey, R. 1971, Phil. Trans. Roy. Soc. Lond.-A 270, 59.

Tousey, R., and Kooman, M. 1971a, Bull. A. A. S. 4, 394.

Tousey, R., and Kooman, M. $\quad 1971$ b, Solar Phys. 21, 401.

Tousey, R., and Brueckner, G. E. 1972, Proc. of the Conf. on Flare-Produced Shock Waves in the Corona, National Center for Atmospheric Research, Boulder, 11-14 Sept., 1972, in press.

Tousey, R., and Kooman, M. 1972, Proc. of the Conf. on Flare-Produced Shock Waves in the Corona, National Center for Atmospheric Research, Boulder, 11-14 Sept., 1972, in press.

Traub, W., and Roesler, F. L. $\quad$ 1971, Astrophys. J. 163, 629.

Triska, P., and Lastovicka, J. 1970, Solar Phys. 15, 504.

Trottet, G. $1971 \mathrm{a}$, Comptes Rendus 272, 286.

Trottet, G. $\quad 1971$ b, Comptes Rendus 272, 1218.

Tucker, W. H., and Koren, M. 1971a, Astrophys. J. 168, 283.

Tucker, W. H., and Koren, M. 1971b, Astrophys. J. 170, 621.

Turiel, I., and MacGregor, G. A. 1970, Astrophys. J. 162, 287.

Turon, P. J., and Lena, P. J. $\quad$ 1970, Solar Phys. 14, 112.

Uchida, Y. 1971, Publ. Astron. Soc. Japan 22, 341.

Uchida, Y., Altschuler, M. D., and Newkirk, G. 1973, Solar Phys. 28, 495.

Unti, T. W. J., Atkinson, G., Wu, C.-S., and Neugebauer, M. 1972 , J. Geophys. Res. 77, 2250.

Vaiana, G. C., and Giacconi, R. 1969, in Plasma Instabilities in Astrophysics, Wentzel and Tidman, eds. (Gordon and Breach).

Vainshtein, L. A., and Safronova, U. I. $\quad$ 1971, Astron. Zh. 15, 223.

Van Speybroeck, L. P., Krieger, A. S., and Vaiana, G. S. $\quad$ 1970, Nature 227, 818.

Vasiljev, B. N., Grineva, Y. I., Zhitnik, I. A., Karev, V. I., Korneer, V. V., Krutov, V. V., and Mandelshtam, S. L. $\quad 1971$, Paper presented at XIV Plenary Meeting of COSPAR.

Vassilyeva, G. Y., Kuznetsov, D. A., and Shpitalnaya, A. A. $\quad$ 1972, Sol. Dann. 2, 99.

Veeder, G. J., and Zirin, H. $\quad$ 1970, Solar Phys. 12, 391.

Velinov, P. I., Dorman, L. I., and Nesterov, G. T. $\quad$ 1970, Ak. Nauk. SSSR Izv. Ser. Fiz., 2183.

Vladimirsky, B. M., and Levitsky, L. S. $\quad$ 1970, Izv. Crimean Astrophys. Obs. 41, 240.

Vorpahl, J. 1972, Solar Phys. 26, 397.

Vorpahl, J., and Pope, T. 1972, Solar Phys. 25, 347.

Vrabec, D. 1971, IAU Symp. 43, p. 329.

Waldmeier, M. 1972, Astr. Mitt. Eidgen. Sternwarte Zurich, 309.

Walker, A. B. C., Jr. $\quad$ 1972, Space Sci. Rev. 13, 672.

Walker, A. B. C., and Rugge, H. R. $\quad 1970$, Astron. Astrophys. 5, 4.

Walker, A. B. C., and Rugge, H. R. $\quad 1971$, Astrophys. J. 164, 181.

Wang, J. R., Fisk, L. A., and Lin, R. P. 1971, Proc. of the XII International Cosmic Ray Conferences Hobart, Sol 438.

Weaver, A. D. 1971, Veterinary Record 89, 288.

Webber, J. C. $\quad$ 1971, Solar Phys. 16, 340.

Wende, C. D. $\quad$ 1972, Solar Phys. 22, 492.

Weiss, N. O. 1971, IAU Symp. 43, p. 757.

White, K. P., III, and Janssens, T. J. 1970, Solar Phys. 11, 291.

Wittman, A. 1971, Solar Phys. 20, 365.

Wiehr, E. $\quad$ 1972, Solar Phys. $24,129$.

Wilcox, J. M. 1971a, IAU Symp. 43, p. 744.

Wilcox, J. M. $\quad 1971$ b, Publ. Astron. Soc, Pac. 83, 561.

Wilcox, J. M. 1972, SUIPR Rept. 469.

Wilcox, J. M., and Colburn, D. S. $\quad$ 1970, J. Geophys. Res. 75, 6366.

Wilcox, J. M., and Gonzalez, W. 1971, Science 173, 820.

Wilcox, J. M., and Colburn, D. S. $\quad 1972$, J. Geophys. Res. 77, 751.

Wilcox, J. M., and Scherrer, P. H. 1972, J. Geophys. Res. 77, 5385.

Wilhjelm, J., and Friis-Christensen, E. 1972, Planet. Space Sci.

Wilson, P. R. 1971a, IAU Symp. 42, p. 475.

Wilson, P. R. $\quad$ 1971b, Solar Phys. 21, 101.

Wilson, P. R. $\quad 1972$, Solar Phys. 22, 434.

Wiseman, M., and Dennison, P. A. $\quad$ 1972, Proc. Astron. Soc. Australia 2, 79. 
Withbroe, G. L. $\quad$ 1971, Solar Phys. 18, 458.

Withbroe, G. L., Dupree, A. K., Goldberg, L., Huber, M. C. E., Noyes, R. W., Parkinson, W. H., and Reeves, E. M. 1971, Solar Phys. 21, 272.

Wöhl, H. 1972, Solar Phys. 24, 342.

Wöhl, H., Wittman, A., and Schröter, E. H. $\quad$ 1970, Solar Phys. 13, 104.

Wood, A. T., Jr., Noyes, R. W., Dupree, A. K., Huber, M. C. E., Parkinson, W. H., Reeves, E. M., and Withbroe, G. L. $\quad$ 1972, Solar Phys. 24, 169.

Wood, A. T., and Noyes, R. W. 1972, Solar Phys. 24, 180.

Yakovkin, N. A., and Zel'dina, M. Yu. 1971, Solar Phys. 19, 414.

Yeh, T., and Axford, W. I. 1970, J. Plasma Phys. 4, 207.

Yoshimura, H. 1971, Solar Phys. 18, 417.

Yoshimura, H., Tanaka, K., Shimizu, M., and Hiei, E. $\quad$ 1971, Publ. Astron. Soc. Japan 23, 443.

Yun, H. S. 1970, Astrophys. J. 162, 975.

Yun, H. S. 1971a, Solar Phys. 16, 379.

Yun, H. S. 1971b, Solar Phys, 16, 398.

Zhugzhda, Y. D. 1971, Cosmic Electrodyn. 2, 253.

Zirin, H. $\quad 1971$, IAU Symp. 43, p. 237.

Zirin, H. 1972, Solar Phys. 22, 34.

Zirin, H., Pruss, G., and Vorpahl, J. 1971, Solar Phys. 19, 463.

Zirker, J. B. 1971, in Physics of the Solar Corona, Macris, ed., p. 140 (Reidel, Dordrecht).

Zvereva, A. M., and Severny, A. B. $\quad$ 1970, Izv. Krymsk. Astrofiz. Obs. 41-42, 97.

Zwaan, C., and Buurman, J. $\quad$ 1971, IAU Symp. 43, p. 220.

SOLAR RADIO ASTRONOMY*

D. J. McLean

\section{General}

The English translation of Zheleznyakov's comprehensive book Radio Emission of the Sun and Planets appeared in 1970. A new review of 'the radio emission from travelling disturbances in the corona - type III, type II and moving type IV bursts...' has recently been published (Wild and Smerd, 1972). A review of the earlier data from the Culgoora radioheliograph will be found in Wild (1970).

Publications which have not been included individually in the references but which are of general interest can be found in the proceedings of the conferences of the Committee of European Solar Radio Astronomers (CESRA) (editors Fokker, Abrami) and Physics of the Solar Corona (editor Macris, 1971). The latter includes a review of space radio-astronomy observations by Papagiannis (1971).

\section{Spectrograph observations}

Spectrograph observations up to kilometre wavelengths are discussed under space radio astronomy.

Radio spectrograph observations continue to bear fruit. At decimetre wavelengths using a highly sensitive, differentiating spectrograph Slottje (1972) has observed a great variety of micro structures during a type IV event, and Rosenberg (1972) has attempted to explain some of them. The occurrence of striking examples of a previously known phenomenon - regular pulsations in type IV events - has stimulated theoretical interest (Rosenberg 1970; McLean et al., 1971; Abrami 1970, 1972) and the publication of more observational data (Gotwols, 1972; Kai, 1973; Caroubalos et al. 1973).

At metre wavelenghts Markeev and Chernov (1970) have also seen unusual bursts and Tarnstrom and Phillips (1972) have made a high-resolution study of what they call spike bursts.

Boischot et al. (1971) have studied the discontinuity in storm activity at about the boundary between

* This note was received too late to be included in the final Commission Report. 